\title{
Contrafreeloading as a function of early environmental rearing conditions
}

\author{
STEPHEN F. DAVIS, BARBARA G. BEIGHLEY, JOHN S. LIBRETTO, \\ and MARY NELL MOLLENHOUR \\ Austin Peay State University, Clarksville, Tennessee 37040 \\ and \\ ROBERT E. PRYTULA \\ Middle Tennessee State University, Murfreesboro, Tennessee 37130
}

\begin{abstract}
Four groups of rat subjects were reared for 45 days in one of four environments (two enriched, a normal, and a deprived, respectively). Following the rearing period, all subjects were administered an 11-day training/testing session consisting of: (1) exposure to free food, (2) barpress training, and (3) choice between obtaining food freely or via barpressing. The results indicated that subjects reared in the deprived environment preferred to barpress significantly more than all other groups. Attention is directed toward a consideration of manipulatory behavior in explaining these results.
\end{abstract}

Influential publications by Jensen (1963) and Neuringer (1969) have indicated, somewhat surprisingly, that under some conditions animals may prefer to perform an. operant task in order to obtain reinforcement rather than simply "freeload." Subsequent investigations have concerned themselves with an identification of those variables influencing this "contrafreeloading" phenomenon. For example, Tarte and Snyder (1972) reported that preference for barpressing was positively related to the length of deprivation. More recently, it has been suggested by Tarte, Towrisend, and Vernon (1973) that the type of rearing environment may have a direct bearing on preference for barpressing. These investigators found that rat subjects reared in stimulus-enriched and stimulus-deprived conditions barpressed significantly less than did subjects reared in motor-enriched and control (standard laboratory cage) conditions. Tarte et al. (1973) suggested that differences in exploratory behavior were responsible for these results. More specifically, it was proposed that the greater the similarity between rearing conditions and the testing situation the greater the exploratory behavior (i.e., barpressing) that would be shown in the testing situation.

The present experiment was designed to further investigate the relationship between early environmental rearing conditions and the contrafreeloading phenomenon. To accomplish this, four types of early rearing environments were employed: (1) enriched similar-containing features similar to the testing

This research was sponsored by a Tower Fund Research Grant from Austin Peay State University to the first author and a Faculty Research Grant from Middle Tennessee State University to the last author. situation, (2) enriched different-featúres similar to testing situation omitted, (3) normal-standard rearing conditions, and (4) deprived-restricted conditions.

\section{METHOD}

\section{Subjects}

Thirty-six male albino rats purchased from the Holtzman Company, Madison, Wisconsin, served as subjects. Upon receipt from the shipper, the subjects were randomly assigned to four equal groups (ES, enriched similar; ED, enriched different; N, normal; and $\mathrm{D}$, deprived) and placed into the respective rearing environments. All subjects were 21 days old upon arrival at the laboratory.

\section{Ápparatus}

Rearing environments. The rearing environment for Group ES consisted of a $183 \times 122 \times 30 \mathrm{~cm}$ plywood box having a $1.27-\mathrm{cm}$ hinged hardware-cloth top. One-half of the floor was also $1.27-\mathrm{cm}$ hardware cloth, with the remainder of the floor being plywood. A sandpile approximately $15 \mathrm{~cm}$ high covered a portion of the plywood floor. Novelty objects, distributed randomly throughout the environment, consisted of eight wooden blocks, five small plastic balls, five small plastic toys, three "tunnels" made of tin cans, a wire exercise wheel, and a cube-shaped plastic "Habbittrail." Three water bottles were attached to one side of the box, and food was placed randomly in the environment. Eight pieces of metal, similar in shape to the operant lever found in the testing chamber, were secured to the walls of the box $10.16 \mathrm{~cm}$ from the floor.The inside walls and plywood portion of the floor were painted gloss white to approximate the interior of the chamber in which testing was conducted. The rearing environment for Group ED was identical, with the following exceptions: (1) the pieces of metal resembling the operant lever were omitted, and (2) the inside walls and floor were not painted.

The rearing environment for subjects in Group D consisted of an adaptation of the standard $(18 \times 18 \times 24 \mathrm{~cm})$ Wahmann laboratory rat cage. A piece of plywood divided each cage in half lengthwise, forming two side-by-side cubicles $(18 \times 9 \times 24 \mathrm{~cm})$. Purina Lab Chow was pulverized to restrict food manipulation and placed in jars which were attached to the front of each cubicle. Water was available on an ad-lib basis in each cubicle. Subjects in Group D were housed one to a cubicle. 
Subjects in Group $\mathbf{N}$ were reared two to a cage in standard Wahmann laboratory cages. Purina Lab Chow bricks were available, as was water, on an ad-lib basis during the rearing period.

Testing situation. All training and testing took place in a standard operant-conditioning chamber $(26 \times 23.80 \times 26.04 \mathrm{~cm})$ which was placed within a sound-attenuating ventilated chamber. Free food was made available on appropriate training and testing days via a metal dish secured to the right rear portion of the operant chamber.

\section{Procedure}

All subjects remained in the respective rearing environments for 45 days. During this period, subjects in Groups ES and ED were frequently handled by the experimenters. Once placed in their rearing cages, subjects in Groups $\mathbf{N}$ and $\mathrm{D}$ were never handled, except during training and testing sessions. On Day 46, all subjects were color coded for identification purposes and placed on a 23-h food-deprivation schedule. On Days 52-54, all subjects were individually placed into the operant chamber and allowed access to the free-food dish (containing $25045-\mathrm{mg}$ pellets) for $30 \mathrm{~min}$. The operant lever was removed on these days. On Days 55-60, all subjects received barpress training for $30 \mathrm{~min} /$ day. Free food was not available on these days. Days 61 and 62 constituted "choice" or testing days. On these days, $45-\mathrm{mg}$ pellets could be "earned" on an FR 1 schedule via barpressing or taken from the free-food dish. The number of pellets obtained freely and by barpressing was recorded for each subject on the 2 choice days.

\section{RESULTS AND DISCUSSION}

Figure 1 shows the mean percent barpress responding for the four groups on the 2 choice days. Analysis of variance performed on the percent barpress data yielded a significant, $F(3,32)=3.19$, $\mathrm{p}<.05$, groups effect. The Newman-Keuls procedure was employed to further investigate this significant effect and indicated that Group D was significantly $(p<.05)$ superior to all other groups which, in turn, did not differ significantly from each other.

As can be seen from Figure 1, the most striking feature of the present study is the significantly elevated barpressing performance of the Group D (deprived) subjects on the 2 choice days.Obviously, these results are somewhat inconsistent with those reported by Tarte et al. (1973). The performance of the stimulus-enriched subjects (Groups ES and ED) in the present experiment is similar to that reported by Tarte et al. (1973); however, a reversal in the performance of the deprived and normal subjects occurred between the two studies. In attempting to resolve discrepant data, one is traditionally bound to consider methodological differences, and several do exist in this case. First, the subjects in the present study were confined to the operant chamber for $1 / 2 \mathrm{~h}$, whereas 1 -h sessions were employed by Tarte et al. (1973). Second, and perhaps more important, an extremely small $\mathrm{N}$ was employed in the Tarte et al. (1973) study (i.e., $N=2,4,4$, and 8, respectively, for motor enriched, control, stimulus, deprived, and stimulus enriched groups). On the other hand, an $\mathrm{N}$ of 9 was used for each group in the present study. At this point, it is difficult to ascertain the relative

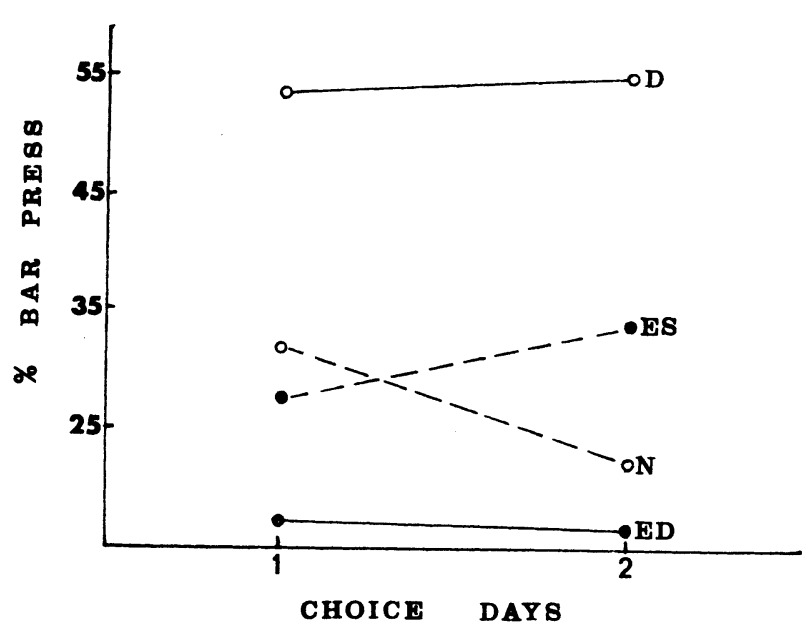

Figure 1. Mean percent barpress on choice days.

contributions of these methodological differences. They do, however, suggest avenues for further research.

Tarte et al. (1973) hypothesized that differences between the rearing environment and exploratory behavior (i.e., barpressing) in the test situation were related. It was further proposed that this relationship could be described by an inverted- $U$ function ranging from stimulus deprived to stimulus enriched. The data from the present study suggests an alternative explanation. It is interesting to note that descriptions (see Bolles, 1967; Fowler, 1965) of "exploratory" behavior have included such terms as locomotion, sniffing, rearing up, etc. Although manipulation is frequently used to describe exploratory behavior, it would appear beneficial to consider it separately or, at lest, as an identifiable component. Viewed in this context, "exploration" would involve behaviors on the part of the subject that would result in movement throughout the environment and consequently result in a decrease in barpressing. On the other hand, a subject engaging in manipulatory behavior might be expected to show an increase in barpressing behavior, especially in the operant-conditioning situation where the operant lever is virtually the only object readily available to be manipulated. Extrapolating to the present study, it is tempting to speculate that the subjects in Group D had a high drive to engage in manipulatory behavior when placed in the testing situation and that this drive was not as pronounced for subjects in Groups ES, ED, and N. The nature of the respective rearing environments is, obviously, supportive of this contention. Subjects in Groups ES, $\mathrm{ED}$, and $\mathrm{N}$ were, in fact, afforded the opportunity to engage in manipulatory behavior prior to being placed in the testing situation, whereas subjects in Group D had literally nothing (not even bricks of food) to manipulate. 


\section{REFERENCES}

Bolles, R. C. Theory of motivation. New York: Harper \& Row, 1967.

Fowler, H. Curiosity and exploratory behavior. New York: Macmillan, 1965.

Jensen, G. D. Preference for barpressing over "freeloading" as a function of number of rewarded presses. Journal of Experimental Psychology, 1963, 65, 451-454.

NEURINGER, A. J. Animals respond for food in the presence of free food. Science, 1969, 166, 399-401.
TARTE, R. D., \& SNYDER, R. L. Barpressing in the presence of free food as a function of food deprivation. Psychonomic Science, 1972, 26, 169-170.

Tarte, R. D., Townsend, S. G., \& Vernon, C. R. Housing environments and the barpressing vs. freeloading phenomenon in rats. Bulletin of the Psychonomic Society, 1973, 2, 79-71.

(Received for publication September 4, 1975.) 